\title{
Monte Carlo Simulation of Ferroelectric Domain Structure and Applied Field Response in Two Dimensions
}

\author{
B.G. Potter, Jr, V. Tikare, and B.A. Tuttle
}

\section{Abstract}

A 2-D, lattice-Monte Carlo approach was developed to simulate ferroelectric domain structure. The model currently utilizes a Hamiltonian for the total energy based only upon electrostatic terms involving dipole-dipole interactions, local polarization gradients and the influence of applied electric fields. The impact of boundary conditions on the domain configurations obtained was also examined. In general, the model exhibits domain structure characteristics consistent with those observed in a tetragonally distorted ferroelectric. The model was also extended to enable the simulation of ferroelectric hysteresis behavior. Simulated hysteresis loops were found to be very similar in appearance to those observed experimentally in actual materials. This qualitative agreement between the simulated hysteresis loop characteristics and real ferroelectric behavior was also confirmed in simulations run over a range of simulation temperatures and applied field frequencies.

\section{Introduction and Motivation}

Ferroelectric oxide ceramics play a critical role in applications that exploit the JUL 131999 broad range of coupled mechanical, electronic and optical phenomena accessible in this class of materials. For example, ferroelectric materials can form the basis for actuator and sensing technologies, non-volatile memory elements, and electro-optical switches and optical phase modulation devices. Thus, the understanding of mechanisms contributing to the intrinsic behavior of these materials is critical to the development of devices with superior performance and high reliability.

It is well understood that the morphology and reorientation dynamics of regions of like-oriented spontaneous polarization (domains) dictate the electrical/mechanical/optical response of a given ferroelectric. Within the ferroelectric phase, the material attempts to attain a domain configuration which minimizes the total energy while satisfying both electrostatic and mechanical boundary conditions. The domain configuration is further complicated in polycrystalline ferroelectric ceramics by the presence of structural discontinuities, e.g. grain boundaries, which can significantly affect the energetics of the domain formation process, influencing both kinetics and final domain assemblage morphology.

The interplay between domain configuration, microstructure and application conditions (e.g. temperature, applied field, pressure) dictate the performance of a given ferroelectric material. Development of a computational simulation technique to predict domain assemblage can contribute to a broader computational modeling scheme which could be used to guide the processing and utilization of ferroelectric ceramics in various applications to optimize performance and evaluate aging effects. 


\section{DISCLAIMER}

This report was prepared as an account of work sponsored by an agency of the United States Government. Neither the United States Government nor any agency thereof, nor any of their employees, make any warranty, express or implied, or assumes any legal liability or responsibility for the accuracy, completeness, or usefulness of any information, apparatus, product, or process disclosed, or represents that its use would not infringe privately owned rights. Reference herein to any specific commercial product, process, or service by trade name, trademark, manufacturer, or otherwise does not necessarily constitute or imply its endorsement, recommendation, or favoring by the United States Government or any agency thereof. The views and opinions of authors expressed herein do not necessarily state or reflect those of the United States Government or any agency thereof. 


\section{DISCLAIMER}

Portions of this document may be illegible in electronic image products. Images are produced from the best available original document. 
Computational modeling of ferroelectric domain characteristics has been the focus of numerous research efforts for a number of years. Techniques including ab initio calculations [1-3], the solution of the Landau-Ginzburg energy relations [4-6], and Isingbased Monte Carlo simulations [7-9] have been utilized with varied degrees of success. The present work develops a lattice-based Monte Carlo simulation technique for the prediction of ferroelectric domain configurations. In its current form, the model is twodimensional and, rather than relying on a Ising or Potts-type Hamiltonian to evaluate neighboring lattice site interactions, the present approach utilizes interaction potentials based on electrostatic phenomena known to occur in ferroelectrics. Future versions of this model will include mechanical and electrostrictive components of the interaction potential.

The present work will describe the model and the influence of simulation conditions and the electrostatic energy terms used, on domain configuration evolution. The collective behavior of the dipole ensemble under an oscillating applied field will also be examined through the simulation of electrical hysteresis loops. The effect of temperature and applied field frequency on the simulated loop behavior will be described.

\section{Model Description \\ Crystal and Domain Representation:}

In a lattice-based Monte Carlo (MC) simulation, the spatial problem domain is discretized into an array, or grid, of lattice points. For the ferroelectric model, each lattice point is assigned the orientation and magnitude of the local, spontaneous dipole moment associated with a single ferroelectric unit cell.

For the purposes of the present study, a tetragonal lattice distortion is assumed to describe the formation of permanent dipoles in the ferroelectric material. If the 2-D simulation lattice is assumed to represent a plane perpendicular to one of the original cubic axes in the parent (paraelectric) crystal, then there are four possible dipole orientations available along two orthogonal basis vectors aligned with the remaining axes of the parent phase crystal. The simulation is further limited to a single crystal orientation only with dipole moment basis vectors aligned with the principal axes of the square lattice used to discretize space. Allowed dipole orientations are therefore up, right, down, and left.

While there is no inherent restriction on the relative orientation and symmetry used for the simulation lattice, the alignment of the simulation lattice basis vectors with those used to represent the dipole moment is consistent with a one-to-one correspondence between a simulation lattice site and a single ferroelectric unit cell and the assumption that crystallographic lattice coherence is maintained through the cubic to tetragonal phase transformation. This choice of lattice and dipole orientation symmetries thus enables the simulation to clearly exhibit the general domain wall orientations and the relative orientations of dipoles in adjacent domains that are anticipated in a tetragonal ferroelectric material (e.g. 180 and 90 degree domain walls).

The dipole moment magnitudes are assumed to be constant during the simulation. In most cases, the local dipole orientations were randomly assigned to lattice points to furnish a starting configuration. Since no elastic strain energy terms (and corresponding 
strain fields) are included in the calculation, the discretization lattice is static throughout the simulation time; there is no motion of adjacent lattice sites with respect to one another.

\section{Interaction Potentials:}

The present work attempts to introduce physically realistic interaction potentials based on the known electrostatic nature of the dipole moment associated with each lattice point. Since the spin states are associated with permanent dipole moments within the ferroelectric, a dipole-dipole potential energy expression has been introduced. Thus, the pairwise interaction potential energy between lattice points is given by the vector expression:

$$
E_{i j}=K \frac{\vec{P}_{i} \cdot \vec{P}_{j}-\left(3\left(\vec{P}_{i} \cdot \hat{n}\right)\left(\vec{P}_{j} \cdot \hat{n}\right)\right)}{r^{3}}
$$

where: $\mathrm{P}=$ dipole moment vector at a given lattice site; $\mathrm{n}=$ unit vector connecting points $i$ and $j ; r=$ magnitude of the separation vector between $i$ and $j ; K=$ scaling factor (contains permittivity of free space).

Ideally, the sum of the dipole-dipole potential energies between all dipoles in the simulation should be used in a calculation of the total simulation potential energy. However, to make the computation tractable, the dipole-dipole potential is truncated at some preset number of lattice units away from the central dipole of interest. This truncation distance is chosen to occur when the magnitude of the potential has dropped to $<2 \%$ of the magnitude at $r=1$ lattice unit. In addition, the truncation distance must also be less than one-half the size of the simulation box to avoid a dipole interacting with its image when using periodic boundary conditions (minimum image convention). While the potential decays at a $1 / r^{3}$ rate, its absolute value depends upon the relative orientations and positions of the dipoles, i.e. the potential is not spherically symmetric (expected for a dipole field). Figure 1 depicts potential curves for two combinations of dipole orientations and relative positions. The truncation distances investigated in the current study are also indicated in the Figure.

It can be seen that the above potential will favor the antiparallel alignment of dipoles on nearest neighbor lattice sites connected by a vector that is normal to the dipole moment vector. Head-to-tail alignment should be favored for nearest neighbor sites connected by a vector parallel to the dipole orientation. It is clear, then that another energy term must be included to begin to form two-dimensional "domains" containing like-oriented spins. Physically, this corresponds to a term based on the polarization gradient between neighboring lattice sites and essentially provide an energy penalty for the formation of such gradients. A candidate domain wall potential, adapted from the expression used by $\mathrm{Hu}$ and Chen [6] was included in the present work. The potential energy density takes the form of: 


$$
D=-J\left\{\begin{array}{l}
\left(\frac{\Delta P_{x}}{\Delta x}\right)^{2}+\left(\frac{\Delta P_{y}}{\Delta y}\right)^{2}+\left(\frac{\Delta P_{x}}{\Delta x}\right)\left(\frac{\Delta P_{y}}{\Delta y}\right)+ \\
\left(\frac{\Delta P_{x}}{\Delta y}+\frac{\Delta P_{y}}{\Delta x}\right)^{2}+\left(\frac{\Delta P_{x}}{\Delta y}-\frac{\Delta P_{y}}{\Delta x}\right)^{2}
\end{array}\right\}
$$

where: $\mathrm{x}$ and $\mathrm{y}$ denote spatial variables, $\mathrm{P}$ is the local electric polarization, and $\mathrm{J}$ is a scaling factor for the domain wall interaction potential. The precise value of $\mathrm{J}$ used in the present work was determined using an approach to be discussed later.

An applied bias field was also included in the simulation. The potential energy of the dipole in the field is expressed as the dot product between the field vector and the dipole moment, as dictated by electrostatics. In the present study, the applied field is taken to be constant over the entire lattice without regard for electrostatic screening effects known to produce local modifications in the field strength. The field-related potential energy is therefore given as:

$$
E_{i}=-\frac{\alpha}{2}\left(P_{i} \cdot F\right)
$$

where: $\mathrm{P}=$ dipole moment, $\mathrm{F}=$ applied field,$\alpha=$ scaling factor.

\section{Boundary Conditions}

Periodic and finite boundary conditions were utilized during simulation runs to investigate the influence of a "surface" on the evolution of the dipole ensemble. As mentioned above, periodic boundaries were imposed using a minimum image convention methodology. The finite boundaries were formed by simple truncation of the simulation lattice. No compensating charges were added to the simulation box "surface." The implications of this approach are discussed below.

\section{Simulation Units}

All simulations were performed in reduced units with length defined by a simulation lattice constant (called a "lattice unit" (LU) ) and the dipole moment defined to be 1 charge-LU. Given the current assignment of a ferroelectric unit cell to each simulation lattice point, the LU is therefore directly related to the crystallographic lattice 
constant. An energy unit (EU) is then equal to the potential energy obtained with two parallel dipole moments of unit magnitude separated by a vector of length one lattice unit oriented perpendicular to the dipole moments. Temperature is reported in terms energy, i.e. kT, in EU's. The temporal unit is a Monte Carlo timestep (MCS). The MC timestep represents $\mathrm{mx} \mathrm{m} \mathrm{MC} \mathrm{move} \mathrm{attempts,} \mathrm{where} \mathrm{m}$ is the size of the simulation box.

\section{Simulation Procedure}

Initially, the model was developed by simulating ensembles using different electrostatic contributions to the total energy. This allowed the effect of each energy term on the dipole ensemble configuration to be studied. Additionally, these preliminary tests allowed an investigation of the simulation strategy as a whole, enabling behavioral artifacts characteristic of the simulation procedure itself to be identified and mitigated prior to further work. During this stage, simulations utilizing the dipole-dipole interaction only were followed by those that added the domain wall potential and then the influence of applied field. Simulation temperatures were chosen typically to provide sufficient thermal energy to allow Monte Carlo move acceptance rates in the range 0.3 to 0.5 , thus favoring evolution of a steady-state dipole configuration within a reasonable computation time without significant randomization of the configuration due to thermal energy. A steady-state condition was defined when little or no change in the ensemble's average total energy was observed with additional MC moves.

After insight regarding the relative impact of the different electrostatic terms and simulation conditions on the evolution of the dipole configuration was evaluated, the model was extended to allow the simulation of ferroelectric-type electrical hysteresis loops. In this case, the applied field value was changed by a known increment and the simulation allowed to evolve from the configuration state developed at the previous field value. The net polarization component aligned with the applied field was calculated from the dipole configuration at each field magnitude. Depending upon the field frequency used (and the computation time available), between 2 and 10 peak-to-peak oscillations in the field were simulated for increased sampling of simulation behavior. The maximum field value was chosen to provide a fully saturated electrical polarization for the longest equilibration time period used. The "effective" field frequency was varied by changing the equilibration time allowed the system at a given field value. Both the effects of field frequency and temperature on the characteristics of the simulated hysteresis loops were investigated in the present study.

\section{$\underline{\text { Results and Discussion }}$}

\section{Dipole-dipole interaction potential only:}

The simulation was first run using only the dipole-dipole electrostatic energy term. A "lock-up" phenomenon was observed in which no change in a dipole orientation could result in a net decrease in total energy. This prevented any further evolution of the 
simulation, effectively arresting the development of the configuration. Lock-up readily occurs at $\mathrm{kT}=0$ and corresponds to a dipole configuration at a local minimum in the energy surface. Similar effects at local minima have been observed in other Potts model simulations, most notably those used for the modeling of grain growth. Using only the dipole-dipole electrostatic potential in the simulation, neither changes in dipole-dipole interaction truncation distance nor lattice symmetry were successful in alleviating the effect. Simulations run under elevated temperature conditions, however, did avoid lockup by providing sufficiently thermal energy to overcome the energy barriers associated with local minima in configuration space.

The effect of temperature on the lock-up phenomenon can be seen in Figure 2 which depicts the total dipole-dipole electrostatic energy of a $20 \times 20$ simulation lattice as a function of Monte Carlo timesteps. When a lock-up condition occurs, there is no additional reduction in the total energy with additional MC timesteps. Introduction of a finite temperature, however, allows the simulation to explore more of configuration space. Note that as the temperature is increased, the "noise" in the curve increased as the simulation accepts a greater range of energetically unfavorable moves.

Figure 3 shows the total energy evolution when different truncation distances are used in the dipole-dipole potential at $\mathrm{kT}=1.5$. It can be seen that the truncation distance does not significantly affect the rate at which the total dipole-dipole energy decreases or the final energy range is attained. As anticipated, examination of the dipole configuration evolution reveals that the actual configurations vary with the truncation distance used, an indication of the highly degenerate nature of the configurational states sampled. The configurations, however, do exhibit the same general characteristics with regard to relative dipole alignments and orientations.

A simulated dipole ensemble at $750 \mathrm{MC}$ timesteps is reproduced in Figure 4 . In general, the configuration consists of single columns or rows of dipoles with the same orientation alternating with columns or rows containing dipoles of antiparallel orientation. A head-to-tail arrangement of dipoles within a given column of the simulation lattice can also be seen in the Figure. These tendencies are predicted by inspection of the dipole-dipole interaction potential in equation 1.

\section{Dipole-dipole + domain wall potential:}

Dipole-dipole interactions alone, with no other energy contribution, produce domains that are essentially one-dimensional in nature, with adjacent domains oriented in antiparallel directions (see Figure 4). With only the dipole-dipole interaction participating, the number of domain walls in an equilibrium energy configuration is extremely high, rivaling the number of domains. These 1-D domain structures are nonphysical and are not consistent with observations of real materials. They are observed in these simulations because no domain wall energy is considered that would favor the formation of larger domains with a shorter total domain wall length.

Figure 5 contains several equilibrium configurations obtained using various values of $\mathrm{J}$ (domain wall potential scaling factor) for $\mathrm{kT}=1.5$ under periodic boundary conditions. As the domain wall increased, through an increased $J$ value, the configurations show a general trend toward more same-orientation neighbors at locations 
perpendicular to the dipole moment orientation. The net effect is to increase the thickness of the domains within the simulation lattice. Note, however, that the tendency toward 180 degree domain boundaries continues through the influence of the dipoledipole interaction.

\section{Dipole-dipole + domain wall + applied field:}

As a final addition to the electrostatically based potential energy for the configuration, the applied field term was included. In general, most simulations resulted in configurations heavily favoring dipole orientations aligned with the field direction, as anticipated. The kinetics of the configuration evolution, however, were dramatically influenced by field strength, with higher field values resulting in a fully aligned configuration in fewer timesteps. The temporal evolution of the dipole configuration in a representative simulation $(\mathrm{kT}=1.5, \alpha=0.25)$ is summarized in Figure 6. Figure 7 depicts temporal evolution of the component of the net dipole moment for the simulation box aligned with the applied field direction for the several applied field strengths.

Evidence for significant retardation in kinetics is observed for $\alpha=0.5$ and 1.0 where the net dipole moment becomes constant (but less than the maximum net dipole moment obtainable) with time. This is expected from previous results for $\mathrm{J}=1.0$ at $\mathrm{kT}=1.5$ (presented above) where the lock-up effect was commonly observed. When the field strength becomes high enough, however, it is sufficient, even at $\mathrm{kT}=1.5$, to overcome local potential energy constraints and allow the configuration to evolve to the fully polarized state.

\section{Effect of Boundary Conditions:}

The simulations described above utilized periodic boundary conditions. Since this implies an infinite (2-D) crystal, it was of interest to investigate the impact of finite boundary conditions upon the behavior of the dipole ensemble. Truncation of the ferroelectric lattice extent is the norm in real materials and the associated depolarization energy is typically identified as the driving force for the formation of polydomain assemblages in fundamental references [10].

To investigate the effect of boundary conditions on the simulated dipole configurations, a simulation employing periodic boundary conditions was compared to simulation results obtained utilizing finite boundaries. In Figure $8 \mathrm{a}$, the final configuration of a periodic boundary condition simulation is shown. Figure $8 \mathrm{~b}$ contains the final configuration (starting from an identical initial configuration) when finite boundary conditions are imposed along only one dimension. The new boundary condition results in a reorientation of dipoles along directions parallel to the finite boundary edges. Note that this is orthogonal to the predominant orientation in the periodic boundary condition run. The introduction of finite boundaries resulted in a final dipole configuration that produces a net zero polarization in the direction of the finite boundaries. A net zero polarization is anticipated along the finite boundary plane normal as the system attempts to minimize the excess electrostatic energy associated with the 
free surface. Interestingly, the system attains the zero polarization condition in this case by making use of the dipole orientations that are available orthogonal to the finite boundary normal. Simulations performed in which only two dipole orientations are available (and aligned with the finite boundary normal) resulted in an assemblage of multiple 180 domains whose net polarization approaches zero through adjustment of domain area. The increased degrees of freedom afforded by the 4 possible dipole orientation directions in the present work, therefore, appears to present a more energetically favorable approach to the reduction in excess electrostatic energy imposed by the finite boundary surfaces.

In the next phase of the study, the finite boundary conditions were imposed along all four edges of the simulation box. Figure $8 \mathrm{c}$ contains the final configuration, again starting from an identical initial dipole configuration to that used in the results of Figure 8a. As the system attempts to minimize the net polarization, now in both vertical and horizontal directions, the dipole ensemble begins to exhibit domain boundaries oriented at a 45 degree angle to the allowed dipole orientations. The dominant dipole orientation change across these boundaries is 90 degrees. The dipoles on either side of these 90 degree domain walls characteristically exhibit a head-to-tail arrangement, dictated by the dipole-dipole electrostatic component of the total energy.It is important to note that the inclusion of finite boundary conditions alone were sufficient to introduce an energetic factor sufficient to favor dipole configurations which minimized the net electric polarization of the ensemble and, in doing so, favor the formation of 90 degree domain walls. In a sense, the simulation implicitly includes an energy component associated with the finite boundary edge that penalizes the simulation for a net polarization. Thus, the excess energy associated with the introduction of finite boundaries is similar in its effect on the evolution of the dipole configuration to an explicitly included depolarization energy.

Minimization of elastic energy is a well-known cause of 90 degree domain formation in tetragonally distorted perovskites. The formation of 90 degree domains in the above simulations, where no mechanical energy is included, should not be interpreted to refute this experimental finding. The simulations do indicate, however, that there can be a contribution to the formation of stable 90 degree domain walls from electrostatic considerations only. In fact, early work by Merz [11] indicates that application of an electric field to single-crystal barium titanate under no external stress can result in 90 degree domain configurations, implying that 90 degree domain boundaries can be generated by electrostatic energy alone.

The energetic implications of the finite boundary conditions were further utilized to develop an empirical value for the domain wall energy scaling factor, J, which would be consistent with phenomenological approaches to the discussion of domain formation based on energy balance between depolarization and domain wall energies. In this case, the total electrostatic energy for a fully aligned dipole ensemble was calculated under both periodic and finite boundary conditions. Reasoning that this configurational state would be unstable with respect to the formation of a polydomain structure under finite boundary conditions, a value for J (the domain wall energy scaling factor) was chosen such that a configuration consisting of a single, 180 degree domain wall traversing the 
simulation box was energetically preferred over the fully aligned configuration. Conversely, the finite $J$ chosen also insured that the single domain configuration was preferred over this two-domain configuration under periodic boundary conditions.

The choice of polydomain configuration for the determination of $\mathrm{J}$ is arbitrary. Polydomain configurations consisting of additional 180 degree domain walls, for example, would result in a lower $\mathbf{J}$ value estimate since the domain wall energy per unit length could be correspondingly lower and still offset the excess energy associated with the free surfaces. The J value developed using the two-domain configuration, therefore, results in an upper bound on the domain wall energy used in the present calculation. While based on a somewhat arbitrary choice of domain configuration, the use of the excess energy determined by the simulation itself to calculate an estimate of $\mathrm{J}$ provides qualitative agreement with the energetics initiating the formation of ferroelectric domains in real materials. This empirical approach within the framework of the present simulation strategy furnishes a reasonable estimate of $J$ and a basis from which to pursue further study. The value of $\mathrm{J}$ found using the above approach was found to be 0.24 and will be used to generate data found in the remainder of this work.

\section{Simulated Electrical Hysteresis}

In an attempt to provide a connection to common, experimentally observed ferroelectric behavior, the modeling approach developed above was adapted to simulate the effect of an oscillating field on the net polarization exhibited by the dipole ensemble, i.e. electrical hysteresis.

The results of the hysteresis simulations run at varied temperatures and effective frequencies are reproduced in Figures 9 and 10, respectively. In Figure 9, all loops were obtained at a frequency requiring $16000 \mathrm{MC}$ timesteps/cycle $\left(6.25 \times 10^{-5}\right.$ cycles/MC timestep). This frequency corresponded to simulation conditions that involved an equilibration time of $200 \mathrm{MCS}$ at each applied field increment. Such a time period under zero field conditions was found to insure equilibration of the simulation potential energy with time. Indeed, no significant change in the hysteresis appearance was observed at half this field frequency (see Figure 10). Hysteresis loops reminiscent of those observed in real, single crystal materials were readily observed in Figure 9. An increase in temperature initially resulted in a decrease in the coercive field followed by reduction in both coercive field and saturated polarization at even greater temperatures until no hysteresis is observed. Again, this general behavior with temperature is consistent with real ferroelectrics as the Curie temperature is approached from below. The simulation behavior is understood in terms of the thermal energy added to the system. As the simulation temperature was increased, the transition probability for energetically unfavorable $\mathrm{MC}$ moves was increased, reducing the time and field strength required to nucleate domains of the required orientation when the field orientation was reversed, thus decreasing the coercive field observed. At even higher temperatures, the thermal energy in the simulation lead to significant disordering of the dipoles, preventing their complete alignment with the field and reducing the observed polarization at a given applied field.

It is important to note that the collapse of the hysteresis loop does not rigorously correspond to a Curie point $(\mathrm{Tc})$ for the simulated material. In the present simulation, the 
local dipole moments always exist; their magnitude is not temperature dependent (as in a real ferroelectric approaching Tc). It is, perhaps, more accurate to refer to the temperature at which hysteresis is lost as a "disordering" temperature to clarify the underlying mechanism causing this behavior. This temperature could then be identified as a phase transition point for the simulated material distinct in nature from that of a real ferroelectric. Interestingly, simulations run over a wide range of frequencies at $\mathrm{kT}=5.85$ appear to consistently reproduce the hysteresis loop observed in Figure 9 at this temperatue, lending support for use of this "order-disorder" transition point as an intrinsic phase boundary for this simulation.

Insight into the dynamics of the simulation are observed in Figure 10 which shows representative hysteresis loops at $\mathrm{kT}=3.35$ using a range of applied field frequencies. As the applied field frequency is increased, the overall area of the hysteresis loop is observed to enlarge, due primarily to a decrease in the coercive field. Of particular interest are higher frequency loops exhibiting greater polarization at $E=0$ than that found at the maximum field. In real ferroelectric measurements, such an appearance is sometimes related to resistive loss in the sample or unavoidable RC-time constant behavior. Here the effect is related to the relative magnitudes of the dipole reorientation rate and the field frequency. At higher frequencies, the simulation does not provide sufficient time for the configuration to "follow" the field, i.e. the time allowed at each applied field increment is insufficent to fully equilibrate the simulation to its lowest potential energy configuration. The situation can be interpreted as a point where the applied field is incremented in a time period that is on the order of the intrinsic realignment time for the individual dipoles in the material. While such applied field frequencies are not generally accessed in the actual ferroelectric measurement, the behavior observed here can provide a link to the timescales prevalent in real materials, providing an opportunity to scale the simulation dynamics accordingly.

\section{Conclusion}

A 2-D, lattice-Monte Carlo approach was developed to simulate the evolution of ferroelectric domain structure. In this model, each simulation lattice point is associated with the magnitude and orientation of the dipole moment of a single ferroelectric unit cell. In its present form, the total energy governing the simulation contains potential energy terms based only on electrostatic interactions between the dipoles themselves and an applied field. The model also utilizes a domain wall energy component that penalizes the simulation for the presence of polarization gradients. An orthogonal set of basis vectors was used to describe 4 possible dipole orientations, consistent with the possible distortion directions of a tetragonally distorted ferroelectric.

Domain configurations were obtained that exhibited 90 and 180 degree domain walls, consistent with the types generally observed in tetragonal ferroelectric crystals. Moreover, an analysis of the influence of boundary conditions indicated that a truncation of the simulation lattice produced an excess potential energy that favored the minimization of net polarization along the direction normal to the finite boundary edges. This result is analogous to the presence of depolarization energy associated with the free surfaces in a real material. Based on an energy balance between the excess energy associated with the finite boundary in the simulation and the energy to required to form a 
polydomain structure that minimizes the net polarization, a scaling factor for the simulated domain wall energy was determined that is consistent with the simulation energetics. This approach is also in qualitative agreement with phenomenological picture of domain formation based on an energy balance between the depolarization and the domain wall energies in real materials.

Finally, the model was extended to allow the simulation of electrical hysteresis as a means of comparison to commonly available ferroelectric measurements. In addition to exhibiting hysteresis loops reminiscent of real materials, the model provided good qualitative agreement with the effects of both temperature and frequency on hysteresis loop appearance.

It is clear that the fidelity of the model will be dramatically increased with its extension to three dimensions and the inclusion of mechanical energy effects. However, even in its current form, the model has been shown to reproduce general domain structure characteristics of actual materials. It also provides a valuable means to evaluate both the influence of electrostatics and boundary type on the equilibrium dipole configuration. Although only localized dipole interaction electrostatics were explicitly included in the model, studies of finite boundary effects indicate that the simulation also captures the excess energy associated with surfaces, a property associated with the overall dipole ensemble and a critical factor in the formation of domains in ferroelectrics.

In addition, relatively straightforward modifications have provided an opportunity to investigate the collective response of the dipole ensemble to static and oscillating applied fields, providing insight into the dynamics of domain switching. The resulting hysteresis loops also furnish a point of comparison to real material behavior enabling verification of the model performance.

Sandia is a multiprogram laboratory operated by Sandia Corporation, a Lockheed Martin Company, for the United States Department of Energy under contract DE-ACO4-94AL $\$ 5060$. 
References:

1. W. Zhong, D. Vanderbilt, and K. M. Rabe, Phys. Rev. Lett. 73 , 1861 (1994).

2. R.E. Cohen and H. Krakauer, Phys. Rev B 42, 6416 (1990).

3. A. Garcia and D. Vanderbilt, Appl. Phys. Lett. 72 , 2981 (1998).

4. W. Cao and L.E. Cross, Phys. Rev. B 44, 5 (1991).

5. S. Nambu and D.A. Sagala, Phys. Rev. B $\underline{50}, 5838$ (1994).

6. H.-L. Hu and L.-Q. Chen, J. Am. Ceram. Soc. 81, 492 (1998).

7. H.M. Duiker and P.D. Beale, Phys. Rev. B 41,490 (1990).

8. M. Abel and R. Siems, Ferroelectrics 126, 275 (1992).

9. J.-M. Liu and Z.G. Liu, Mater. Lett. $\underline{36}, 17$ (1998).

10. for example: M.E. Lines and A. Glass

11. W. Merz, Phys. Rev. 93,690 (1954). 
Figure Captions:

Figure 1: Graphical representation of dipole-dipole interaction potential for selected combinations of dipole orientations.

Figure 2: Total dipole-dipole potential energy obtained for a $20 \times 20$ simulation with MC timestep at different temperatures.

Figure 3: Potential energy evolution with MC timestep for identical simulations using dipole-dipole interaction potentials with varied truncation distances.

Figure 4: Representative configuration after 750 MCS using dipole-dipole interaction only.

Figure 5: Equilibrium configurations obtained from simulations performed using both the dipole-dipole and domain wall potential energy terms; $\mathrm{kT}=1.5$.

Figure 6: Representative simulation results showing the evolution of the configuration under an applied field for $\mathrm{J}=1.0, \mathrm{kT}=1.5, \alpha=0.25$ with both dipole-dipole and domain wall interactions included.

Figure 7: Temporal evolution of the component of the net dipole moment oriented along the applied field direction under different applied field strengths. $(\mathrm{kT}=1.5, \mathrm{~J}=1.0)$.

Figure 8: Representative final configurations showing the effect of boundary conditions on simulation evolution (identical initial starting configurations): a. periodic boundary conditions, all sides; $b$. finite boundary conditions, top and bottom, periodic boundary conditions, left and right; c. finite boundary conditions, all sides.

Figure 9: Representative hysteresis curves simulated at various temperatures. All loops obtained using a frequency of $6.25 \times 10^{-5}$ cycles/MC timestep.

Figure 10: Representative hysteresis curves simulated at various applied field frequencies. All loops obtained at $\mathrm{kT}=3.35$. Applied field frequencies given for each loop in units of cycles/MC timestep. 


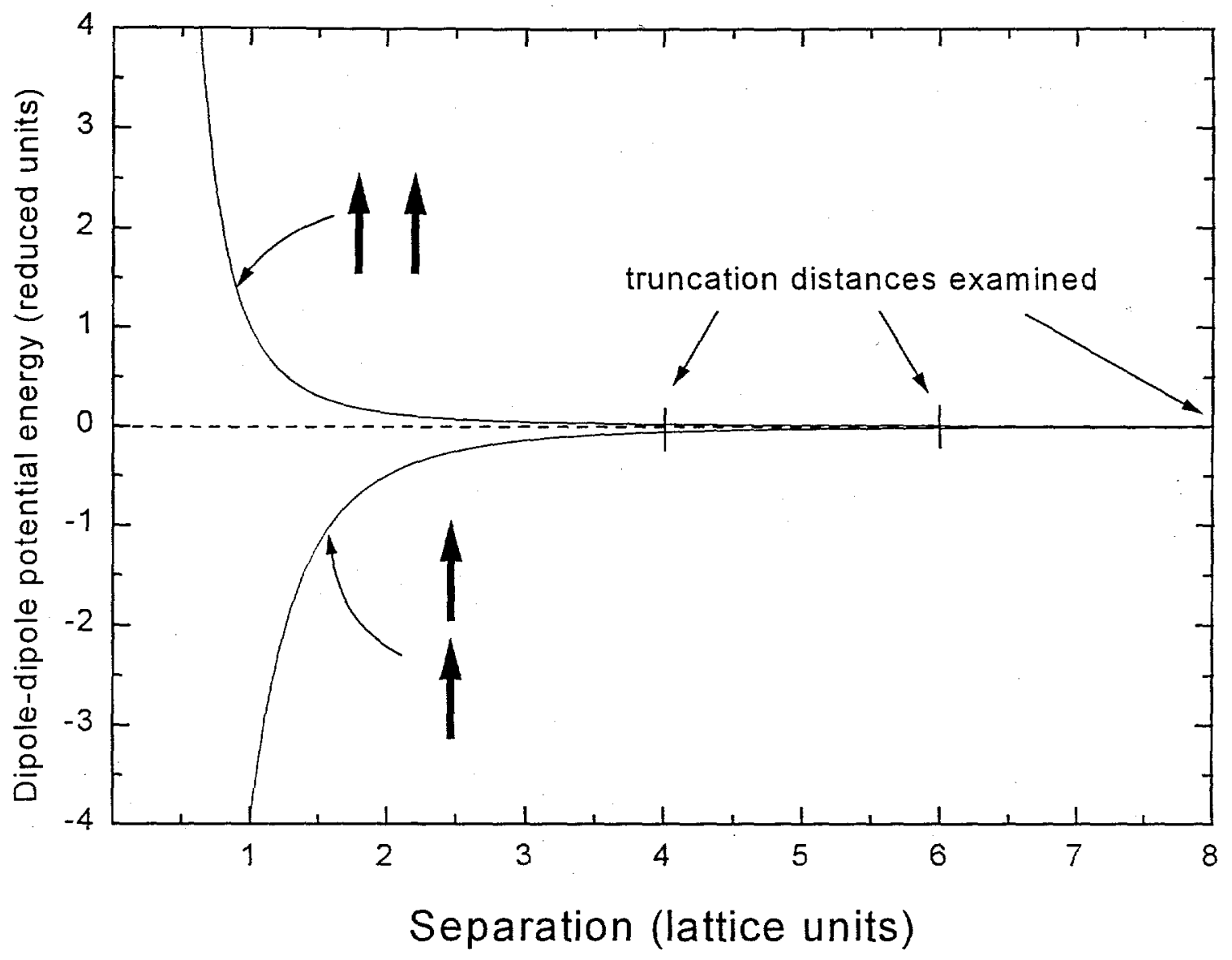

Figure 1 


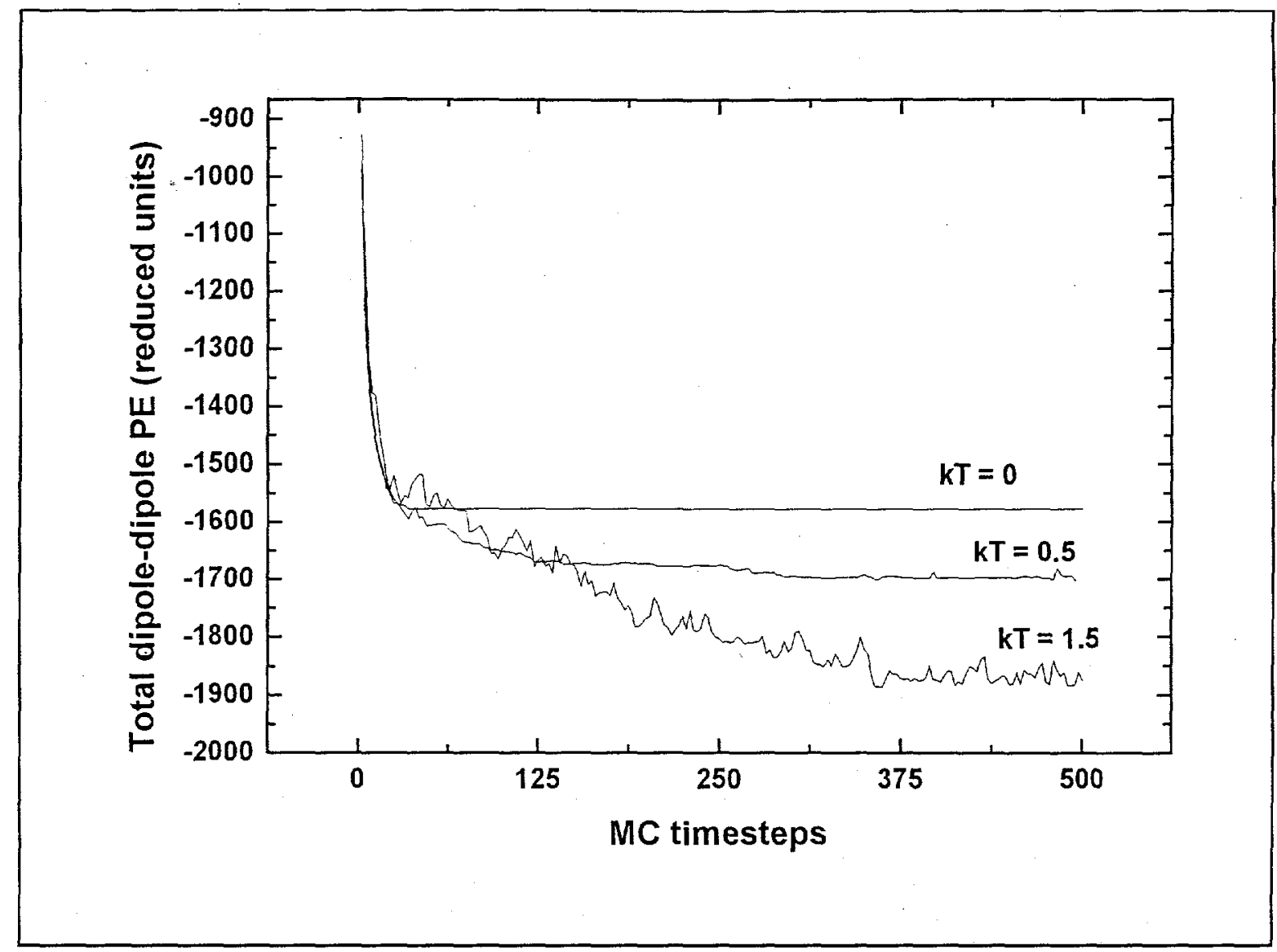

Figure 2 


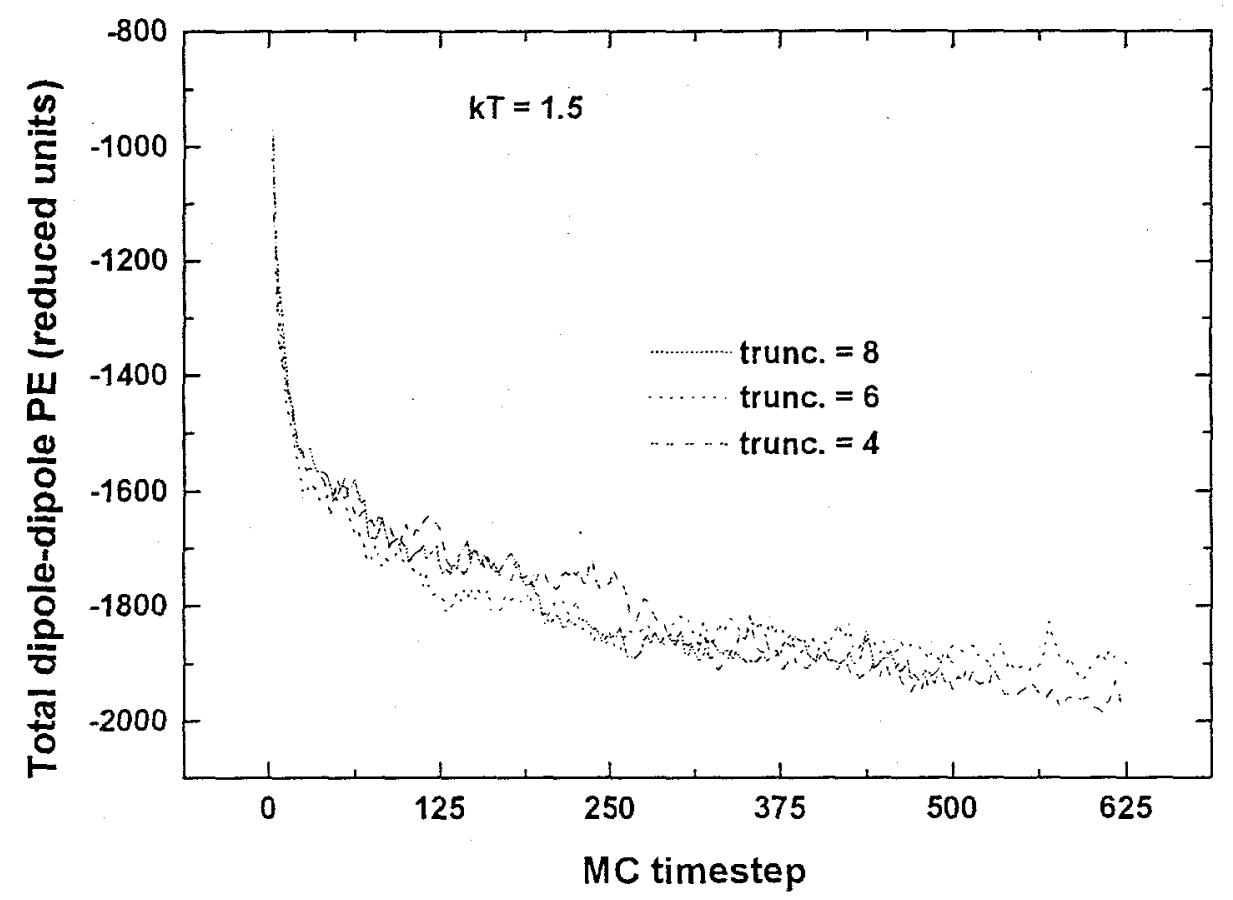

Figure 3 


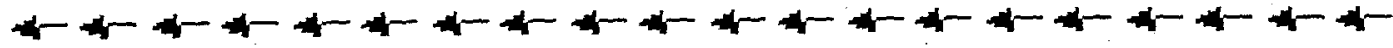

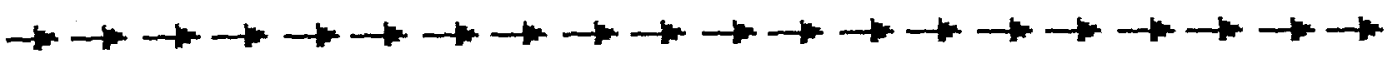

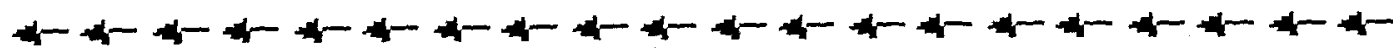

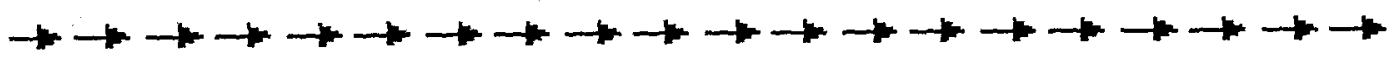

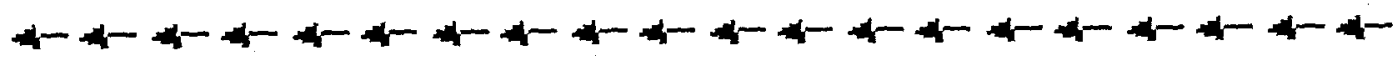

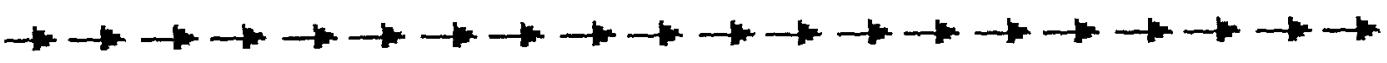

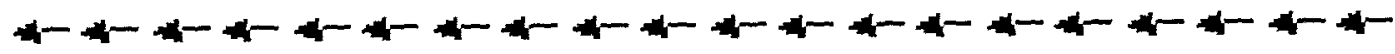

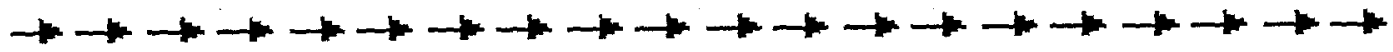

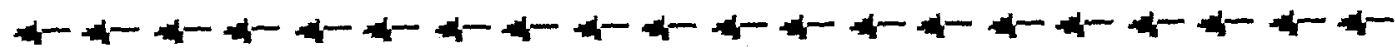

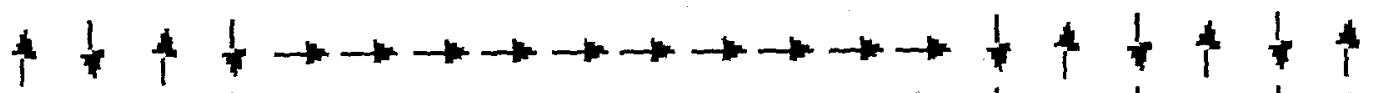

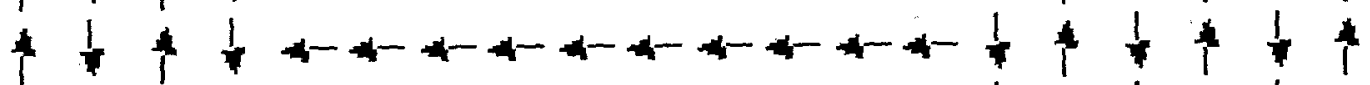

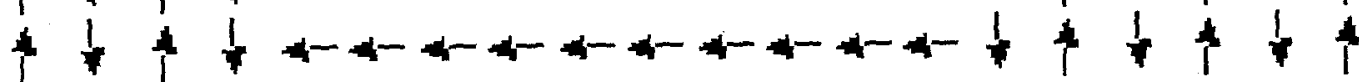

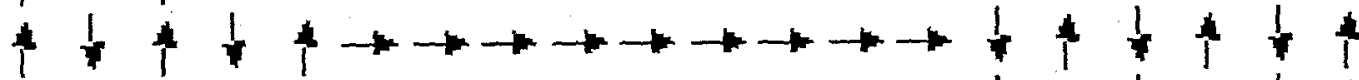

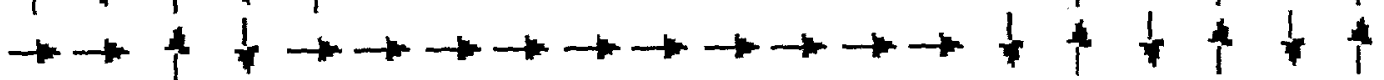

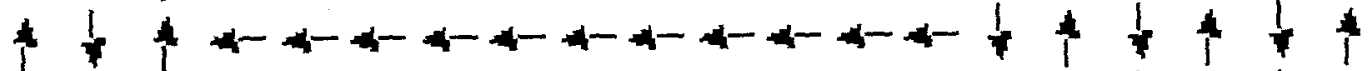

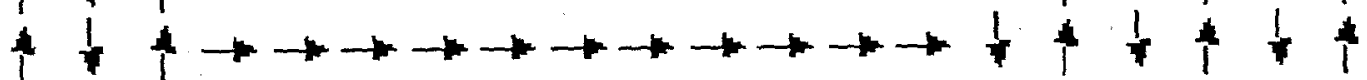

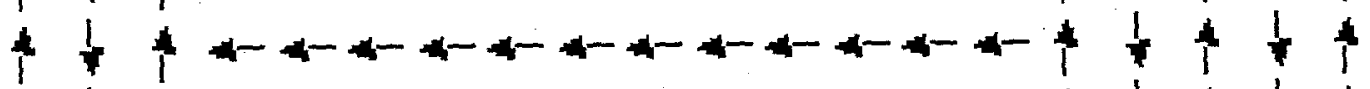

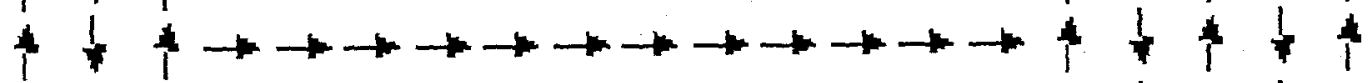

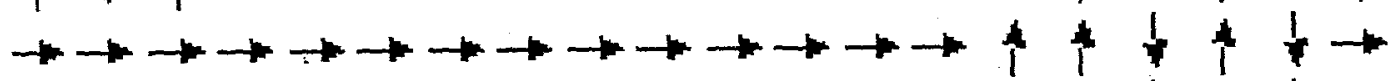

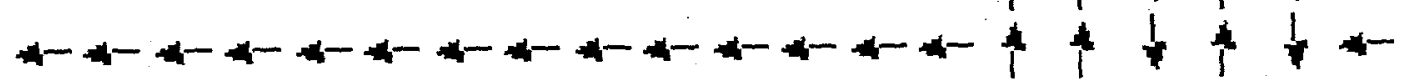

Figure 4 


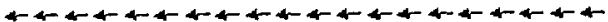
$\rightarrow \rightarrow \rightarrow \rightarrow \rightarrow \rightarrow \rightarrow \rightarrow \rightarrow \rightarrow \rightarrow \rightarrow \rightarrow \rightarrow \rightarrow \rightarrow \rightarrow \rightarrow$

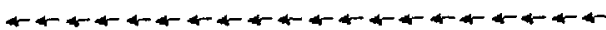
$\rightarrow \rightarrow \rightarrow \rightarrow \rightarrow \rightarrow \rightarrow \rightarrow \rightarrow \rightarrow \rightarrow \rightarrow \rightarrow \rightarrow \rightarrow \rightarrow \rightarrow \rightarrow$

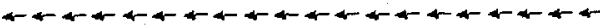
$\rightarrow \rightarrow \rightarrow \rightarrow \rightarrow \rightarrow \rightarrow \rightarrow \rightarrow \rightarrow \rightarrow \rightarrow \rightarrow \rightarrow \rightarrow \rightarrow$ -4ه $\rightarrow \rightarrow \rightarrow \rightarrow \rightarrow \rightarrow \rightarrow \rightarrow \rightarrow \rightarrow \rightarrow \rightarrow \rightarrow \rightarrow \rightarrow \rightarrow \rightarrow$

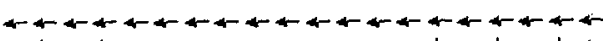
$4|+1 \rightarrow \rightarrow \rightarrow \rightarrow \rightarrow \rightarrow \rightarrow \rightarrow \rightarrow|+1|+1|$

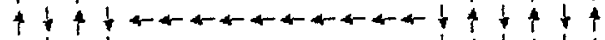
$+1+1+4+\infty \leftarrow+\leftarrow \leftarrow+1+1+1+1$ it $1+1 \rightarrow \rightarrow \rightarrow \rightarrow \rightarrow \rightarrow \rightarrow \rightarrow+1+1+1$ $\rightarrow \rightarrow 1+\rightarrow \rightarrow \rightarrow \rightarrow \rightarrow \rightarrow \rightarrow+1+1+1$

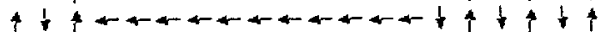
t $1+\rightarrow \rightarrow \rightarrow \rightarrow \rightarrow \rightarrow \rightarrow \rightarrow \rightarrow 1+1 \mid+1$

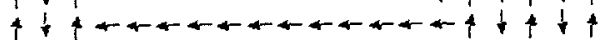
i $1 \rightarrow \rightarrow \rightarrow \rightarrow \rightarrow \rightarrow \rightarrow \rightarrow \rightarrow \rightarrow \rightarrow+1+1+1$ $\rightarrow \rightarrow \rightarrow \rightarrow \rightarrow \rightarrow \rightarrow \rightarrow \rightarrow \rightarrow \rightarrow \rightarrow+1 \frac{1}{1}+\vec{i}$

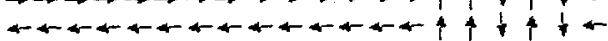

$$
\mathrm{J}=0
$$

$+1+t+1+1+t+1+t+t+1+1$ $+1+b+1+1+1+1+1+1+1+1$ $+1+1+1+1+1+1+t+1+1$ $+1+1+1+1+1+1+1+1+1+1$ $\hat{1}+1+1+1+1+1+1+1+1+1$ $+1+1+1+1+1+1+1+1+1+1$ $+1+t+1+1+1+1+1+1+1$ $+1+1+1+1+1+1+1+1+1+1$

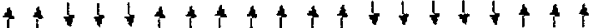
$+1+1+1+1+1+1+1+1+1$ $+1+1+1+1+1+1+1+1+1$ $+1+1+1+1+1+1+1+1+1+$ $+1+1+1+1+1+t+1+1+1$ $+1+1+1+1+1+1+1+1+1+1+1$ $+1+1+1+1+1+1+1+t+1+1$ $+1+1+1+1+1+1+1+1+1$ $+1+1+1+1+1+1+1+1+t+1$ $+1+1+1+1+1+1+1+1+1+1$ $+1+1+1+1+1+1+1+1+1+1$ $+1+1+1+1+1+1+1+t+1+1$

$$
\mathrm{J}=0.6
$$

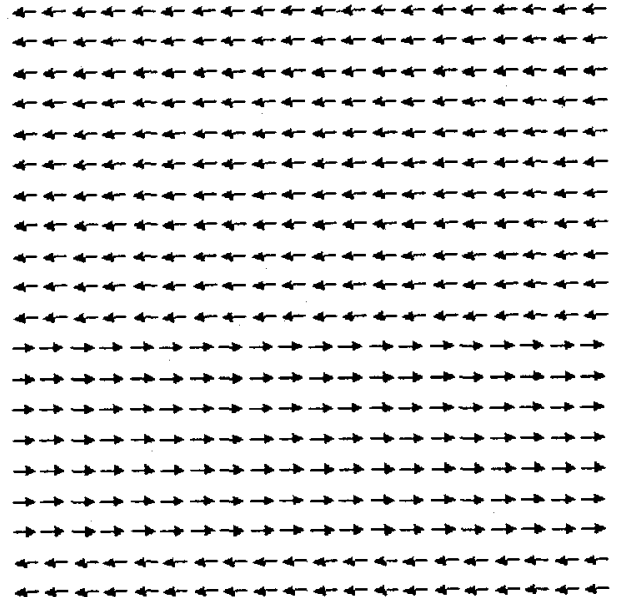

$\mathrm{J}=1.0$

Figure 5 


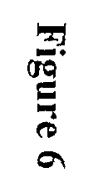
us

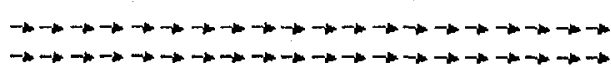
$\rightarrow \rightarrow \rightarrow \rightarrow \rightarrow+\rightarrow \rightarrow+\rightarrow \rightarrow \rightarrow \rightarrow \rightarrow \rightarrow+\infty$ $\rightarrow \rightarrow \rightarrow \rightarrow \rightarrow-\rightarrow \rightarrow \rightarrow \rightarrow-\infty \rightarrow+\rightarrow \rightarrow-\infty \rightarrow+\infty$ $\rightarrow \rightarrow \rightarrow \rightarrow \rightarrow \rightarrow \rightarrow \rightarrow \rightarrow \rightarrow \rightarrow \rightarrow \rightarrow \rightarrow \rightarrow \rightarrow \rightarrow \rightarrow \rightarrow+\infty$ $\rightarrow 11 \rightarrow \rightarrow \rightarrow \rightarrow \rightarrow \rightarrow \rightarrow \rightarrow \rightarrow \rightarrow \rightarrow \rightarrow \rightarrow \rightarrow 1$

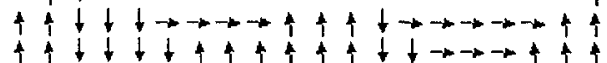

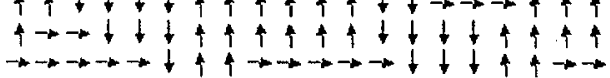
$\rightarrow \rightarrow \rightarrow \rightarrow \rightarrow \rightarrow \rightarrow \rightarrow \rightarrow \rightarrow \rightarrow \rightarrow \rightarrow \rightarrow \rightarrow \rightarrow \rightarrow+\infty$ $\rightarrow \rightarrow \rightarrow \rightarrow \rightarrow \rightarrow \rightarrow \rightarrow \rightarrow-m \rightarrow \rightarrow \rightarrow \rightarrow \rightarrow \rightarrow \rightarrow \rightarrow \rightarrow-\infty$

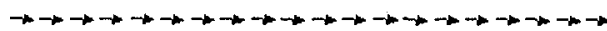
$\rightarrow \rightarrow \rightarrow \rightarrow \rightarrow \rightarrow \rightarrow \rightarrow \rightarrow \rightarrow \rightarrow-\rightarrow \rightarrow \rightarrow \rightarrow \rightarrow-\rightarrow-\rightarrow+\infty$

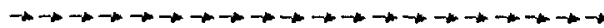

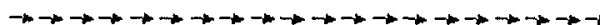
$\rightarrow+\rightarrow+\infty+\infty$ $\rightarrow \rightarrow \rightarrow \rightarrow \rightarrow \rightarrow \rightarrow \rightarrow \rightarrow \rightarrow \rightarrow \rightarrow \rightarrow \rightarrow \rightarrow \rightarrow \rightarrow+\infty$

$\rightarrow \rightarrow \rightarrow \rightarrow \rightarrow \rightarrow \rightarrow \rightarrow \rightarrow \rightarrow \rightarrow \rightarrow \rightarrow \rightarrow \rightarrow \rightarrow \rightarrow \rightarrow+\infty$

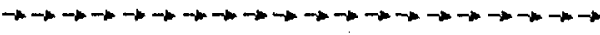
$\rightarrow \rightarrow \rightarrow \rightarrow \rightarrow \rightarrow \rightarrow \rightarrow \rightarrow \rightarrow \rightarrow \rightarrow \rightarrow \rightarrow \rightarrow \rightarrow \rightarrow \rightarrow \rightarrow-\rightarrow$ $\rightarrow \rightarrow \rightarrow \rightarrow \rightarrow \rightarrow \rightarrow \rightarrow \rightarrow \rightarrow \rightarrow \rightarrow \rightarrow \rightarrow \rightarrow-\rightarrow+\infty$ $\rightarrow \rightarrow \rightarrow \rightarrow \rightarrow \rightarrow \rightarrow \rightarrow \rightarrow \rightarrow \rightarrow \rightarrow \rightarrow \rightarrow \rightarrow \rightarrow \rightarrow-\rightarrow$ $\rightarrow \rightarrow \rightarrow \rightarrow \rightarrow \rightarrow \rightarrow \rightarrow-\rightarrow \rightarrow \rightarrow \rightarrow \rightarrow \rightarrow \rightarrow \rightarrow \rightarrow \rightarrow+\infty$ $\rightarrow \rightarrow \rightarrow \rightarrow \rightarrow \rightarrow \rightarrow \rightarrow \rightarrow \rightarrow \rightarrow \rightarrow \rightarrow \rightarrow \rightarrow \rightarrow \rightarrow+\infty$ $\rightarrow \rightarrow \rightarrow \rightarrow \rightarrow \rightarrow \rightarrow \rightarrow \rightarrow \rightarrow \rightarrow \rightarrow \rightarrow \rightarrow \rightarrow \rightarrow \rightarrow \rightarrow \rightarrow-\infty$

$\rightarrow \rightarrow \rightarrow \rightarrow \rightarrow \rightarrow \rightarrow \rightarrow \rightarrow+\infty \rightarrow+\infty \rightarrow+\infty \rightarrow+\infty$

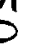

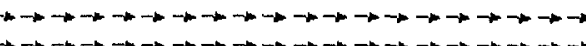

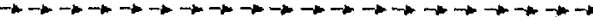
$\rightarrow \rightarrow \rightarrow \rightarrow \rightarrow \rightarrow \rightarrow \rightarrow \rightarrow \rightarrow \rightarrow \rightarrow \rightarrow \rightarrow \rightarrow \rightarrow-\infty$

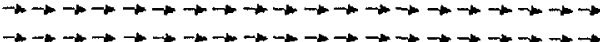
$\rightarrow \rightarrow \rightarrow \rightarrow \rightarrow \rightarrow \rightarrow \rightarrow \rightarrow \rightarrow \rightarrow \rightarrow \rightarrow \rightarrow \rightarrow \rightarrow \rightarrow-\rightarrow \rightarrow+\infty$

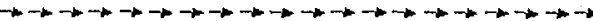
$\rightarrow \rightarrow \rightarrow \rightarrow \rightarrow \rightarrow \rightarrow \rightarrow \rightarrow \rightarrow \rightarrow \rightarrow \rightarrow \rightarrow \rightarrow-\rightarrow+\infty$ $\rightarrow \rightarrow \rightarrow \rightarrow \rightarrow \rightarrow \rightarrow \rightarrow \rightarrow \rightarrow \rightarrow \rightarrow \rightarrow \rightarrow \rightarrow \rightarrow-\rightarrow \rightarrow+\infty$

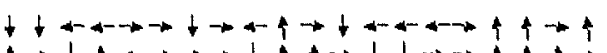
$1 \rightarrow+1 \leftrightarrow \rightarrow \rightarrow+1+\rightarrow 1+\rightarrow \rightarrow+1 \rightarrow$

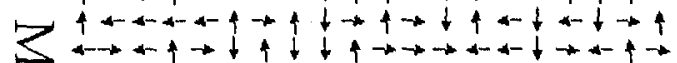

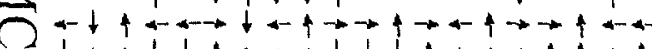
ㄱ. $\downarrow+-1+1 \rightarrow+1+1+1+1 \rightarrow+1+1+1$

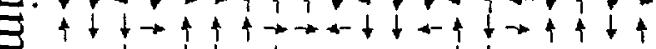
$-1+1+1+\downarrow+1 \rightarrow-1+1+1+1$ $+1+4-1+1+1+4+1+1+1$ $\rightarrow \rightarrow 1+1 \rightarrow+1 \rightarrow 1 \rightarrow 1+1+1<1+1$ $8 \vec{b}+1+1+1+1+1+1+1-1+1$

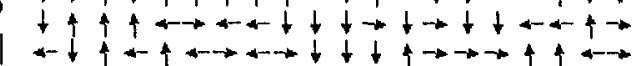

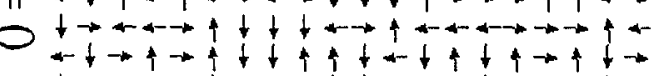

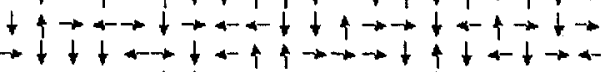

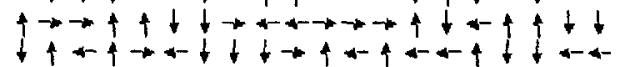

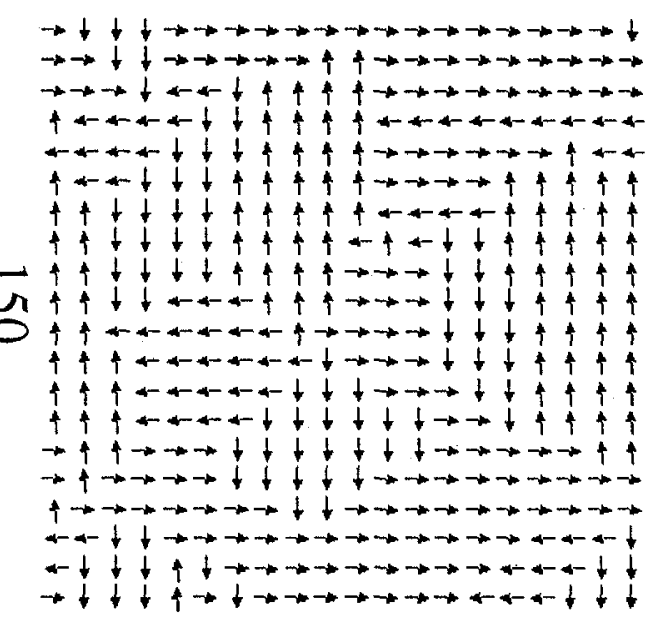

$\rightarrow \rightarrow \rightarrow \rightarrow \rightarrow \rightarrow \rightarrow \rightarrow \rightarrow \rightarrow \rightarrow \rightarrow \rightarrow \rightarrow \rightarrow \rightarrow \rightarrow \rightarrow \rightarrow \rightarrow \rightarrow \rightarrow \rightarrow$

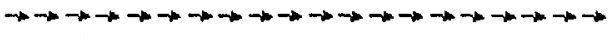
$\rightarrow \rightarrow \rightarrow \rightarrow \rightarrow \rightarrow \rightarrow \rightarrow \rightarrow \rightarrow \rightarrow \rightarrow \rightarrow \rightarrow \rightarrow+\rightarrow \rightarrow \rightarrow \rightarrow+\infty$ $4-4-4-\infty \rightarrow+1 \mid+1+\infty-4-4-4-4-4$ $2-4+1+1+1+1+1+1+4-4$ $1+1+1+1+1+1+1+1+1+1$ $+1+t+1+t+1+t+1+t+1$

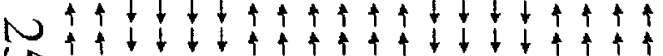
Ut $1+1+1+\infty t+1+1+1+1+1+1$

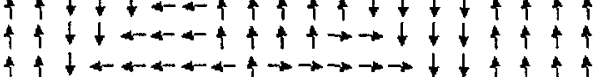

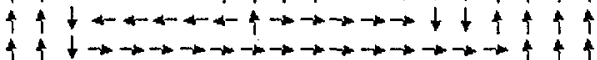
i $1+\rightarrow \rightarrow \rightarrow \rightarrow \rightarrow \rightarrow \rightarrow \rightarrow \rightarrow \rightarrow \rightarrow \rightarrow \rightarrow \rightarrow \rightarrow \rightarrow \rightarrow \rightarrow \rightarrow \rightarrow+\rightarrow$ $\rightarrow \rightarrow \rightarrow \rightarrow \rightarrow \rightarrow \rightarrow \rightarrow \rightarrow \rightarrow \rightarrow \rightarrow \rightarrow-\rightarrow \rightarrow \rightarrow$ $\rightarrow \rightarrow \rightarrow \rightarrow \rightarrow \rightarrow \rightarrow \rightarrow \rightarrow \rightarrow \rightarrow \rightarrow \rightarrow \rightarrow \rightarrow \rightarrow \rightarrow \rightarrow \rightarrow \rightarrow \rightarrow$ $\rightarrow \rightarrow \rightarrow \rightarrow \rightarrow \rightarrow \rightarrow \rightarrow \rightarrow \rightarrow \rightarrow+\infty \rightarrow+\infty \rightarrow+\infty \rightarrow+\infty$

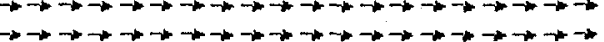




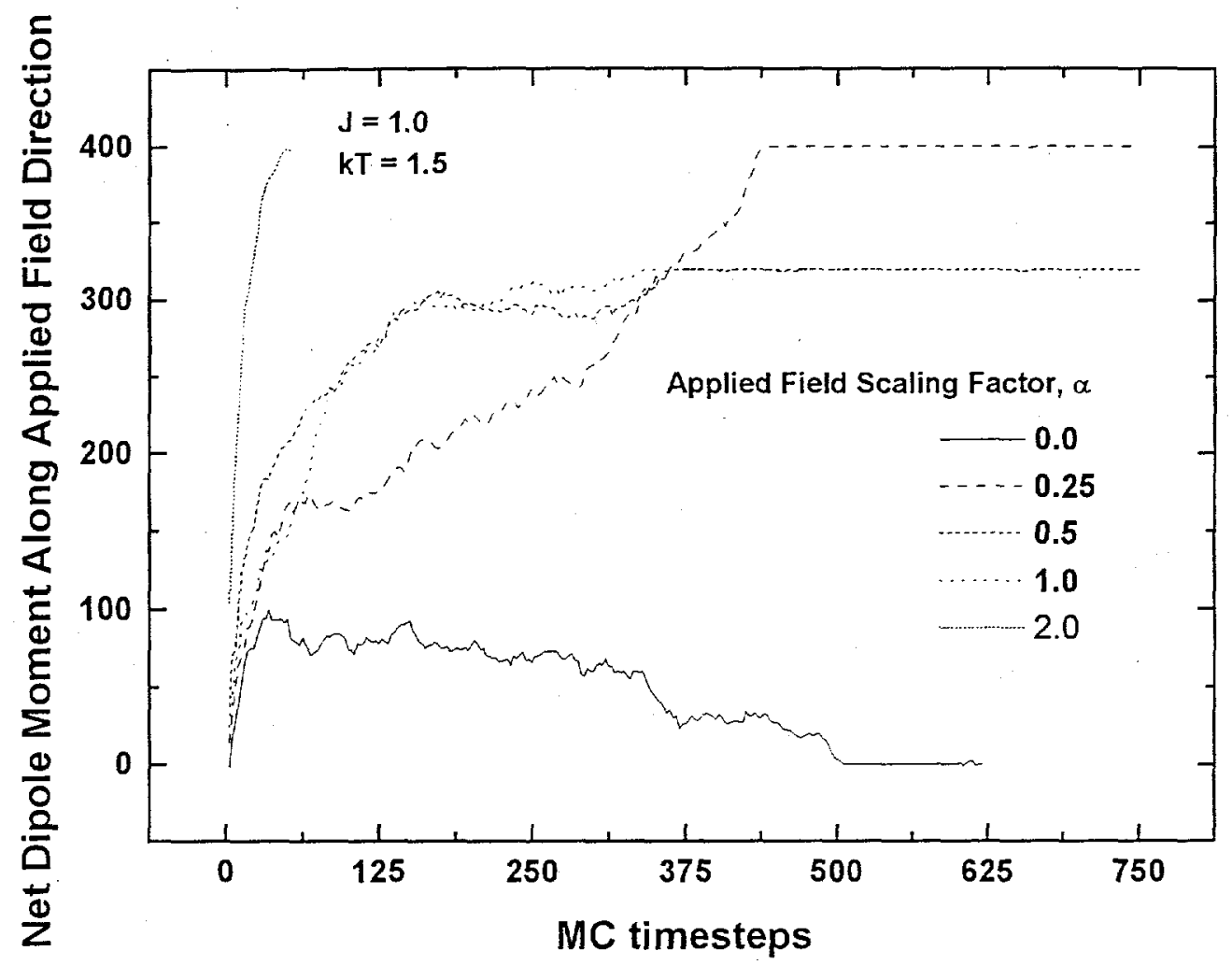

Figure ? 
$+1+1+1+1+1+1+1+4+1+1$ $+4+\{+1+1+1+1+1+1+1$ $-4+4+1+1+1+1+1+1+1+1$ is $+1+1+1+1+1+1+1+1+1$ \& $\{+1+1+1+1+1+1+1+1+1$ t $+1+4+1+1+1+1+1+1+1$ $+4+1+1+1+1+1+1+1+1$ $+1+1+1+1+1+1+1+1+1$ $4+4+4+1+1+1+2-1+1+1$ $+1+1+1+1+1+1+1+1+1$ $+4+1 \rightarrow+1+1+1+1+1+1$ $+1+1+1+1+1+1+1+1+1$ $t+1+1+1+1+1+1+1+1+1$ $+1+1+1+1+1+1+1+1+1$ i $\{+1 \rightarrow+1+1+1+1+1+1+1$ $+t^{+}+1+1+1 \rightarrow+1+1+1+1$ $+\leqslant+1+1+1+1+1+1+1+1$ $\rightarrow+1+1+1+1 \rightarrow 1+1+1 \rightarrow 1+1$ $\rightarrow+1+1+1+1+1+1+1+1+1$ $+4+4+1,1+1,145151$

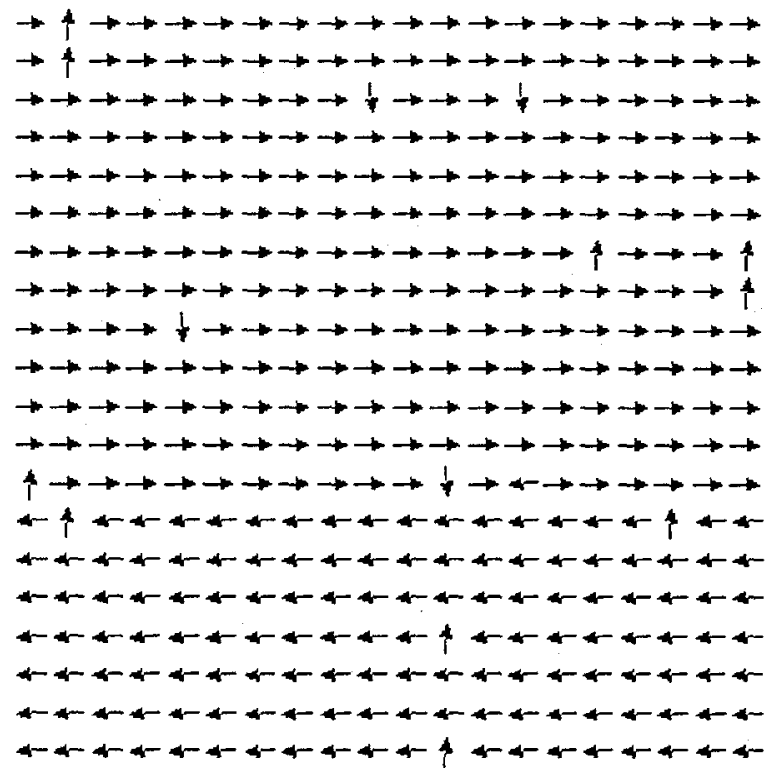

b.

a.

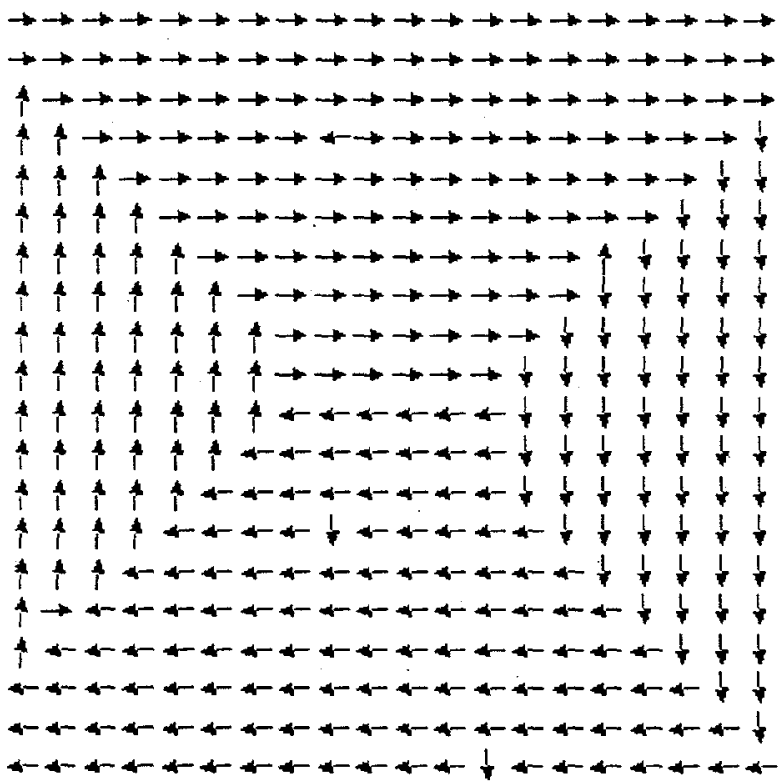

C.

Figure 8 
Monte Carlo Simulation of Ferroelectric....

Potter, 1812

Page 22 of 24
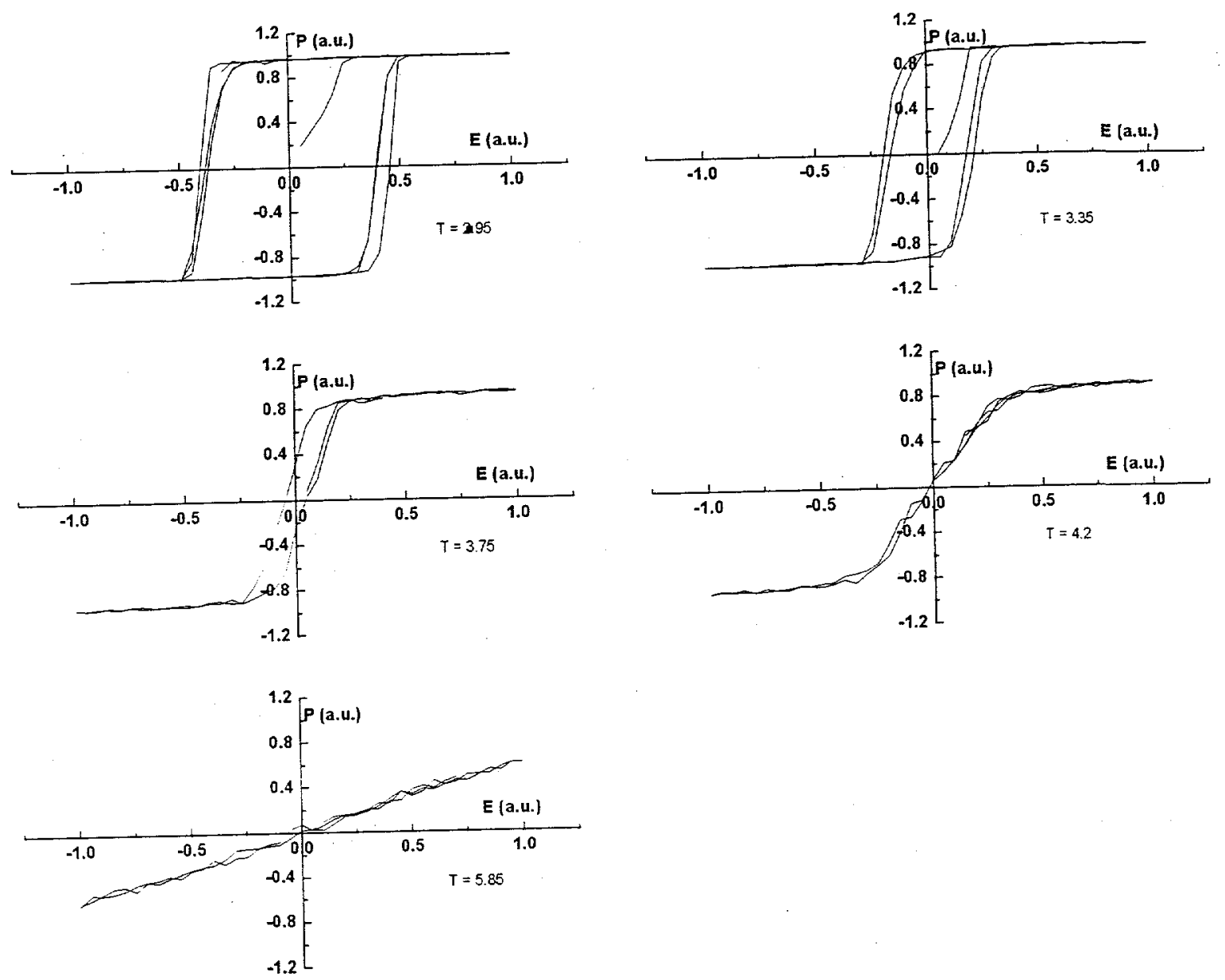

Figure 9 

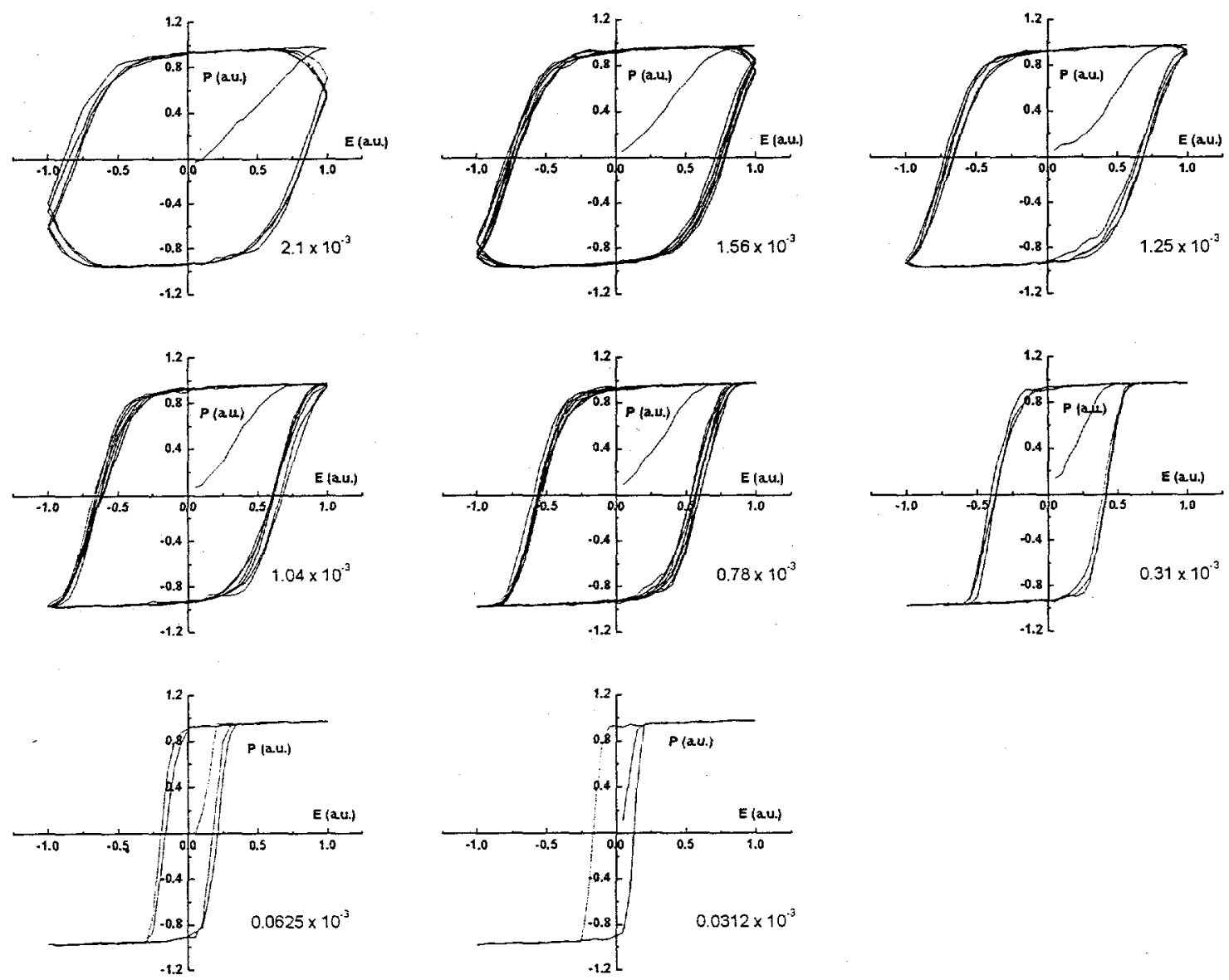

Figure 10 
